\title{
Modified Gravity with Vector Distortion and Cosmological Applications
}

\author{
Jose Beltrán Jiménez $^{1, *}$ and Tomi S. Koivisto ${ }^{2}$ \\ 1 Aix Marseille Univ, Université de Toulon, CNRS, CPT, Marseille 13288, France \\ 2 Nordita, KTH Royal Institute of Technology and Stockholm University, Roslagstullsbacken 23, \\ SE-10691 Stockholm, Sweden; tomik@astro.uio.no \\ * Correspondence: jose.beltran@cpt.univ-mrs.fr
}

Academic Editors: Mariusz P. Dąbrowski, Manuel Krämer and Vincenzo Salzano Received: 7 April 2017; Accepted: 19 May 2017; Published: 24 May 2017

\begin{abstract}
We briefly review the basics of Weyl geometry and its natural extension by a general linear "distortion" of the metric connection by a vector field. A special class of the connections has torsion but retains the Weyl's semi-metricity condition. We present ghost-free gravitational theories in this geometrical setup and highlight their possible cosmological applications, such as new self-tuning solutions and new bouncing solutions found in the quadratic-curvature theories. The vector distortion can mimic the cosmological effects of dark matter.
\end{abstract}

Keywords: modified gravity; Weyl geometry; cosmology

\section{Introduction}

General Relativity (GR) provides a beautiful description of the gravitational interaction within the framework of Riemannian geometry, as the curvature of the spacetime. A crucial assumption in the traditional description of GR is the identification of the connection with the Christoffel symbol of the metric, which therefore also determines the affine structure of the spacetime. However, in geometry, this structure is a priori independent, and much of its richness is lost by stipulating the connection as the Christoffel symbol of the metric. In physics, on the other hand, one usually regards connections as fundamental dynamical objects (e.g., in Yang-Mills theories), so that depriving it of independent dynamics in the case of gravity can jeopardise its gauge interpretation in the usual manner. Thus, one is naturally led to consider more general connections also in the description of the gravitational interaction.

Remarkably, the Einstein-Hilbert action with an independent connection is still equivalent to GR because the Levi-Civita solution is recovered dynamically for the connection (up to an irrelevant projective mode). However, more general actions with an independent connection lead to different physical theories than with the metric connection [1,2], and the new effects reveal a geometrical interpretation in terms of non-metricity and torsion [3-7]. An approach that lies between the traditional metric restriction and unleashing the full affine connection (which in four dimensions has 64 components), is to let the geometry go non-Riemannian only in specifically motivated forms. An outstanding instance of this approach is the Weyl geometry [8] where a vector field is added to the connection to make it invariant under local rescalings of the metric when the vector field simultaneously transforms as a $U(1)$ gauge field. If the (effective) action is not homogeneous of order 0 in the metric, however, the invariance is broken (unless one introduces additional ingredients). In general then, the theory can have scales and the vector field in the connection participates in the dynamics. Interesting aspects of such theories have been considered recently, e.g., [9-11]. In this note, we will introduce a simple extension of the Weyl connection and discuss gravitational theories that can be consistently formulated within the extended class of geometries. The generic quadratic-curvature 
action will be shown to reduce to a four-parameter vector-tensor theory that suggests a variety of implementations in cosmology.

\section{Geometry with Vector Distortion}

In Weyl's extension of the Riemannian geometry, the metric-compatibility condition is replaced by the so-called semi-metricity condition, $\hat{\nabla}_{\mu} g_{\alpha \beta}=-2 A_{\mu} g_{\alpha \beta}$ where $A_{\mu}$ is a new vector field introduced to realise an invariance under a rescaling (usually called Weyl transformation) $g_{\mu \nu} \rightarrow e^{2 \Lambda(x)} g_{\mu v}$ with the simultaneous $U(1)$ transformation $A_{\mu} \rightarrow A_{\mu}-\partial_{\mu} \Lambda(x)$. If we assume vanishing torsion, this new condition can be easily solved to obtain the connection in the usual way as

$$
\hat{\Gamma}_{\beta \gamma}^{\alpha}=\Gamma_{\beta \gamma}^{\alpha}-\left(A^{\alpha} g_{\beta \gamma}-2 A_{\left(\beta_{\gamma} \delta^{\alpha}\right.}^{\alpha}\right)
$$

where $\Gamma_{\beta \gamma}^{\alpha}$ is the Levi-Civita connection. The vector field enters in such a way that the aforementioned Weyl transformation leaves the connection invariant. This in turn means that the Riemann tensor of this connection will also exhibit the Weyl invariance. If we momentarily forget about this invariance, the connection (1) calls for a natural extension to include all the possible terms linear in the vector field and with no derivatives, i.e., the Weyl connection can be extended to [12]

$$
\hat{\Gamma}_{\beta \gamma}^{\alpha}=\Gamma_{\beta \gamma}^{\alpha}-b_{1} A^{\alpha} g_{\beta \gamma}+b_{2} \delta_{(\beta}^{\alpha} A_{\gamma)}+b_{3} \delta_{[\beta}^{\alpha} A_{\gamma]}
$$

with $b_{i}$ some constant parameters. The difference between this connection and the Levi-Civita connection goes under the name of distortion and, in the present case, it contains both non-metricity and torsion.

The analogous of the (in-)compatibility condition for the metric can be expressed as

$$
\hat{\nabla}_{\mu} g_{\alpha \beta}=\left(b_{3}-b_{2}\right) A_{\mu} g_{\alpha \beta}+\left(2 b_{1}-b_{2}-b_{3}\right) A_{(\alpha} g_{\beta) \mu} .
$$

A remarkable case within this family of theories is $2 b_{1}-b_{2}-b_{3}=0$, that makes Equation (3) invariant under Weyl transformations. Thus, this particular case gives a generalisation of the Weyl geometries where the connection also contains a torsion piece given by the same Weyl vector. The extended geometries defined by Equation (2) will be our starting point to construct gravitational theories, and we will see that the semi-metricity of the connection emerges as a crucial and rather generic requirement for viable theories.

Let us note that in the context of metric-affine gauge theory, many of the exact solutions that have been obtained in the past, would be described by an affine connection of the form (2). These "triplet" solutions, which have only excited the vector modes, that are also all proportional to each other in the three distinct sectors of geometry, have been mainly obtained for spherically symmetric ansätze, see [13] for a summary. The triplet feature of those solutions was probably due to the difficulty of finding more general exact solutions in metric-affine gauge theories, which, nevertheless, may demonstrate the relevance of the simple parameterisation in the study of more elaborated theories. Here, we take the connection in the form (2) to begin with, and aim to find cosmological solutions.

Before entering into the construction of the gravitational actions, let us introduce the key geometrical object, namely the Riemann tensor defined as

$$
\mathcal{R}_{\mu \nu \rho}^{\alpha} \equiv \partial_{\nu} \hat{\Gamma}_{\mu \rho}^{\alpha}-\partial_{\mu} \hat{\Gamma}_{v \rho}^{\alpha}+\hat{\Gamma}_{\nu \lambda}^{\alpha} \hat{\Gamma}_{\mu \rho}^{\lambda}-\hat{\Gamma}_{\mu \lambda}^{\alpha} \hat{\Gamma}_{v \rho}^{v}
$$

From this object, we can define three different traces: the usual Ricci tensor $\mathcal{R}_{\mu v} \equiv \mathcal{R}_{\mu \alpha v}{ }^{\alpha}$, the co-Ricci tensor $\mathcal{P}_{\mu}{ }^{\alpha} \equiv g^{v \rho} \mathcal{R}_{\mu v \rho}{ }^{\alpha}$ and the homothetic tensor $\mathcal{Q}_{\mu v} \equiv \mathcal{R}_{\mu v \alpha}{ }^{\alpha}$. 


\section{Gravitational Lagrangians with Vector Distortion}

In this section, we will discuss the theories that arise when considering general Lagrangians depending on the curvature invariants associated with the vector-distorted connection. We review two classes of gravitational theories, given by the $f(\mathcal{R})$ actions and the most general (even-parity) quadratic curvature actions, and end by briefly commenting on the cubic curvature actions.

\section{1. $f(\mathcal{R})$ Theories}

We start by considering the popular theories described by an arbitrary function of the Ricci scalar. Our connection with vector distortion yields

$$
\mathcal{R}=R-\beta_{1} A^{2}+\beta_{2} \nabla \cdot A,
$$

where we have defined $\beta_{1} \equiv-3\left[4 b_{1}^{2}-8 b_{1}\left(b_{2}+b_{3}\right)+\left(b_{2}+b_{3}\right)^{2}\right] / 4$ and $\beta_{2} \equiv-3\left(2 b_{1}+b_{2}+b_{3}\right) / 2$ and $R$ denotes the Ricci scalar of the Levi-Civita part of the connection. Now, considering a Lagrangian of the type $f(\mathcal{R})$, we straightforwardly see that the field equations impose the vector field to be a pure gradient. This means that, despite having a vector field, the theory only contains one scalar propagating dof. The usual approach to these theories reveals that the Lagrangian can be expressed in the equivalent form [12]

$$
\mathcal{L}=\varphi R+\frac{\beta_{2}^{2}}{4 \beta_{1} \varphi} \partial_{\mu} \varphi \partial^{\mu} \varphi-V(\varphi)
$$

with $\varphi$ the mentioned scalar dof and $V(\varphi)$ a potential whose form depends on the original function $f$. It is now apparent that we recover a Brans-Dicke theory. Interestingly, the semi-metric geometry with $b_{3}=2 b_{1}-b_{2}$ highlighted above gives $\beta_{2}^{2}=6 \beta_{1}$ and the resulting theory describes a non-propagating scalar field, similar to the case of $f(\mathcal{R})$ theories within an affine approach [1,2]. The reason why the scalar field does not propagate is that the kinetic term can be fully absorbed by performing a conformal transformation and, thus, the scalar field only appears as an auxiliary field. If we consider a quadratic function, the Einstein frame description of the theory is given by

$$
\mathcal{L}=\frac{M_{p l}^{2}}{2} \tilde{R}-\frac{1}{2} \tilde{g}^{\mu l} \tilde{\phi}_{, \mu} \tilde{\phi}_{, v}-\frac{3}{4} M_{p l}^{2} M^{2}\left(1-e^{-\sqrt{\frac{2}{3 \alpha}} \frac{\tilde{\phi}}{M_{p l}}}\right)^{2}
$$

where $M^{2}$ stands for the new mass scale introduced and we have defined $\alpha \equiv 1-\beta_{2}^{2} /\left(6 \beta_{1}\right)$. We have thus obtained the one-parameter family of potentials that precisely corresponds to the generalisation of the Starobinsky model $(\alpha=1)$ dubbed the $\alpha$-attractor [14]. In [15], this result was also obtained by considering gravitational actions with auxiliary vector fields, whose relation with broken scale symmetry has then been considered further $[16,17]$.

\subsection{Quadratic Actions}

The previous section has shown that theories based on the Ricci scalar associated to connections with vector distortion reduce to scalar-tensor theories of the Brans-Dicke type. Obtaining actual vector-tensor theories requires going beyond the Ricci scalar to actions with more general geometrical objects. A natural family of theories to contemplate is the class of Lagrangians that are quadratic in curvature invariants. Let us then write down the most general quadratic action and $D$-dimensions and respecting a parity symmetry 


$$
\begin{aligned}
\mathcal{S}= & \mu \int \mathrm{d}^{D} x \sqrt{-g}\left[\mathcal{R}^{2}+\mathcal{R}_{\alpha \beta \gamma \delta}\left(d_{1} \mathcal{R}^{\alpha \beta \gamma \delta}+d_{2} \mathcal{R}^{\gamma \delta \alpha \beta}-d_{3} \mathcal{R}^{\alpha \beta \delta \gamma}\right)\right. \\
& -4\left(c_{1} \mathcal{R}_{\mu \nu} \mathcal{R}^{\mu v}+c_{2} \mathcal{R}_{\mu v} \mathcal{R}^{v \mu}+\mathcal{P}_{\mu \nu}\left(c_{3} \mathcal{P}^{\mu v}+c_{4} \mathcal{P}^{v \mu}-c_{5} \mathcal{R}^{\mu \nu}-c_{6} \mathcal{R}^{v \mu}\right)\right. \\
& \left.\left.+\mathcal{Q}_{\mu v}\left(c_{7} \mathcal{Q}^{\mu v}+c_{8} \mathcal{R}^{\mu v}+c_{9} \mathcal{P}^{\mu v}\right)\right)\right] .
\end{aligned}
$$

with $\mu$ a parameter of dimension $4-D$ and $d_{i}, c_{i}$ some dimensionless constants. To avoid ghosts associated to higher than second-order field equations, it is crucial that the theory should reduce to the usual Gauss-Bonnet term when the connection reduces to the Levi-Civita one, i.e., for $A_{\mu}=0$. This imposes the following constraints for the parameters

$$
d_{1}+d_{2}+d_{3}=\sum_{i=1}^{6} c_{i}=1
$$

We can now express everything in terms of curvatures of the Levi-Civita connection and the vector field as follows $[12,18]$ :

$$
\mathcal{L}_{(2)}=-\frac{1}{4} F_{\mu v} F^{\mu v}+\xi A^{2} \nabla \cdot A-\lambda A^{4}-\beta G^{\mu v} A_{\mu} A_{v}+\gamma_{1}(\nabla \cdot A)^{2}+\left(\gamma_{2} A^{2}+\gamma_{3} \nabla \cdot A\right) R
$$

where $F_{\mu \nu}=\partial_{\mu} A_{v}-\partial_{\nu} A_{\mu}$ is the usual field strength and the parameters $\xi, \lambda, \beta$ and $\gamma_{i}$ are given in terms of the original parameters appearing in (8). Although we have been careful to guarantee that, in the absence of the vector distortion, the theory is healthy, we see that the vector field in general propagates four dofs due to the presence of kinetic terms breaking the $U(1)$ gauge invariance so that all four components of the vector field will be dynamical, one of which will be associated to an Ostrogradski instability. Another way of seeing this pathology more clearly is by introducing a Stückelberg field $\chi$ to restore the $U(1)$ symmetry via the replacement $A_{\mu} \rightarrow A_{\mu}+\partial_{\mu} \chi$ so that the $\gamma_{1}$-term $(\nabla \cdot A)^{2}$ will give rise to the higher order derivative term $(\square \phi)^{2}$ that, again, will be responsible for the Ostrogradski instability. Similarly, the non-minimal derivative couplings with $\gamma_{2}$ and $\gamma_{3}$ will lead to the presence of ghosts. In order to render the theory stable, we thus need to impose that $\gamma_{1}=\gamma_{2}=\gamma_{3}=0$. Remarkably, these conditions lead directly to the class of semi-metric, or generalised Weyl geometries with $b_{3}=2 b_{1}-b_{2}$, discussed above (and in [18] in more detail). Thus, non-trivial quadratic-curvature theories exist only for the semi-metric class of geometries. For completeness, we also include the usual Einstein-Hilbert term so that the final vector-tensor theory reads

$$
\mathcal{L}_{(2)}=\frac{1}{2} M_{\mathrm{Pl}}^{2} R-\frac{1}{4} F_{\mu v} F^{\mu v}+\frac{1}{2} M^{2} A^{2}-\lambda A^{4}+\xi A^{2} \nabla \cdot A-\beta G^{\mu v} A_{\mu} A_{\nu} .
$$

where the Einstein-Hilbert term has additionally provided the vector field with a mass $M$. This action was obtained for the case of purely Weyl geometry in [11], but the result can be extended to the whole family of generalised Weyl geometries. One important feature of these theories is that $\xi$, $\lambda$ and $\beta$ are actually proportional to $D-4$ so that they vanish in four dimensions. In that case, the theory reduces to a simple Proca field whose mass is parametrically given by the Planck mass. This would make it an ideal candidate for dark matter, as suggested in [11]. Because its mass is typically very large, the isotropy theorem of [19-21] naturally applies for the vector.

In order to obtain more general theories, a suitable limit of the original parameters together with $D \rightarrow 4$ should be taken. In that case, the $\xi$-term describes a vector Galileon interaction, which represents one of the healthy non-gauge invariant derivative self-interactions for a vector field [22-25]. Similarly, the $\beta$-term is the only healthy coupling of the vector field to the curvature. The coupling to the Einstein tensor could be relevant in the generation of cosmic magnetic fields [26], since the coupling could provide a dynamical explanation for the old Schuster-Blackett phenomenological law that states a proportionality between the angular momentum and the magnetic moment of astrophysical objects. 
The cosmological phenomenology of the full four-parameter action (11) was studied in [18]. The very rich phenomenology offered by these theories can be traced to the non-dynamical nature of $A_{0}$. This feature allows it to be algebraically solved in terms of $H$ and, when plugged into the Friedman equation, the resulting cosmology is driven by an effective Friedman equation with a non-linear source depending on the energy density of the present matter (other than the vector). More explicitly, we eventually obtain an equation of the form $F\left(H^{2}, \rho\right)=0$, with $F$ some non-trivial function, that determines the homogeneous cosmology (see Figure 1). Isotropic de Sitter solutions are common attractor solutions in these theories, so they could naturally give cosmologies with dynamical dark energy. In fact, for some choices of the parameters, the scale of the de Sitter solution is determined by the theory parameters and is insensitive to the presence of a cosmological term. A minimal example model is given by erasing the two last terms from (11): the effect of the vector distortion then reduces to nothing but a cosmological constant (in the isotropic background). The model given by erasing instead the middle two terms can realise self-tuning cosmologies, so that Minkowski is a solution even if a cosmological constant is present. Another characteristic feature of these theories is the existence of cosmologies with a sudden singularity where the derivative of the Hubble expansion rate diverges, but the energy density and the Hubble parameter remain finite. Finally, the general theories (11) can also accommodate non-singular bouncing cosmologies without a violation of the null energy condition, or a re-collapsing phase without a spatial curvature. In Figure 1, we show the phase space of two specific models where some of the discussed features are shown. Let us finally note that the coupling to the Einstein tensor will lead to the generation of an anomalous propagation speed of gravitational waves whenever around a non-trivial background of $A_{\mu}$. In these cases, the parameter $\beta$ will be subject to the constraints obtained in [27] from the Hulse-Taylor binary pulsar.
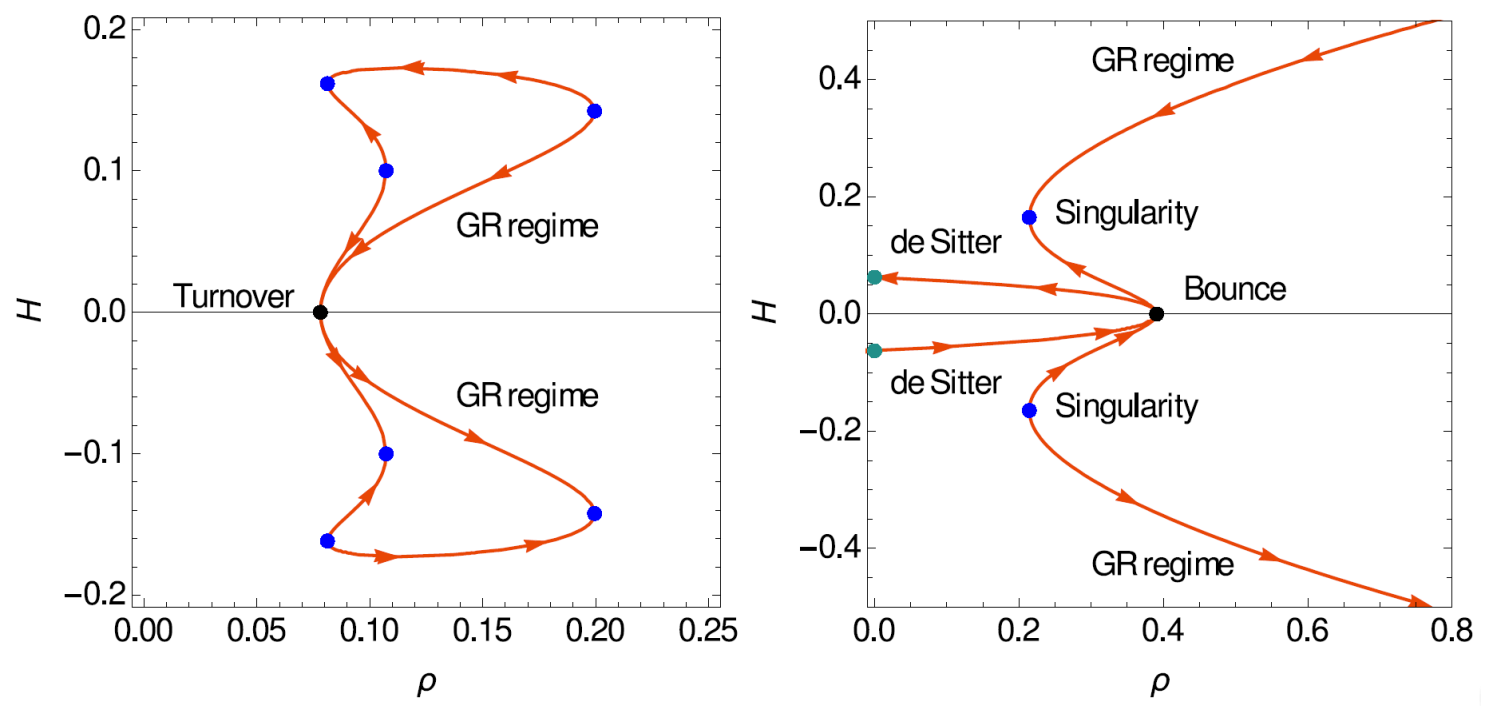

Figure 1. In this figure, we show the cosmological evolution for two specific examples of the vector-tensor theory described by (11). The left panel corresponds to $\beta=3, \lambda=0.2, \xi=1 / 3$ and $M^{2}=0.5 M_{\mathrm{Pl}}^{2}$ and the right panel to $\beta=0, \lambda=1, \xi=6$ and $M^{2}=2.5 M_{\mathrm{Pl}}^{2}$. The arrows indicate the flow generated by the time evolution. The blue points are the finite- $H$ sudden singularities mentioned in the main text, and the black points correspond to bouncing and re-collapsing universes. We also show the de Sitter solutions that can be both attractor or repeller solutions. An interesting property of the models, such as the one shown in the left panel, is the compactness of the phase space so that both $\rho$ and $H$ are bounded. 


\subsection{Cubic Theories}

The theories constructed out of quadratic curvature terms give rise to an interesting class of vector-tensor theories (11), but care had to be taken to sort out the healthy ones. In four dimensions, the Gauss-Bonnet already saturates the Lovelock series of allowed healthy actions for the Levi-Civita connection, and so one might expect that no healthy interactions for the connection with vector distortion can be constructed. However, we can use the result found by Horndeski [28], that second-order field equations result from the non-minimal coupling $L^{\mu v \alpha \beta} F_{\mu \nu} F_{\alpha \beta}$ with $L^{\mu \nu \alpha \beta}=-\frac{1}{2} \epsilon^{\mu \nu \rho \sigma} \epsilon^{\alpha \beta \gamma \delta} R_{\rho \sigma \gamma \delta}$ the double dual Riemann tensor. This motivates consideration of cubic terms involving the double dual Riemann tensor. It was shown in $[11,18]$ that these terms give rise to the following vector-tensor interactions:

$$
\begin{aligned}
\mathcal{L}_{(3)}= & L^{\mu \nu \alpha \beta} F_{\mu v} F_{\alpha \beta}+2\left(2 b_{1}+b_{2}+b_{3}\right) \tilde{F}^{\mu \alpha} \tilde{F}^{v}{ }_{\alpha} \nabla_{\mu} A_{v} \\
& +\frac{1}{2}\left[\left(2 b_{1}-b_{2}-b_{3}\right)^{2} A^{2} g^{\mu v}-2\left(4 b_{1}^{2}+\left(b_{2}+b_{3}\right)^{2}\right) A^{\mu} A^{v}\right] F_{\mu \alpha} F_{v}{ }^{\alpha} .
\end{aligned}
$$

The first line is the aforementioned Horndeski vector-tensor interaction plus another non-gauge invariant derivative self-interaction for the vector field. The second line has more standard harmless self-interactions for the vector field. The cosmology of the pure Horndeski interaction was studied in [29] and in [30], where it was shown that the Horndeski term typically introduces pathologies whenever it dominates. The effects of the additional terms appearing in (12) remain to be studied and it is possible that the pathologies found in the pure Horndeski case could be alleviated in the general case.

\section{Conclusions}

A vector distortion of the affine connection provides a natural geometric framework wherein to interpret existing vector-tensor theories and to derive consistent novel theories. The case of the $f(\mathcal{R})$ theory, where the vector effectively reduces to a scalar dof, presents one of the possible geometrical realisations of the $\alpha$-attractor generalisation of the Starobinsky inflation. We have also reviewed the perhaps more interesting theories that are obtained by considering generic quadratic and cubic actions in the curvature. These theories show a very rich cosmological phenomenology, including de Sitter phases, bounces, re-collapsing universes without spatial curvature, and self-tuned solutions. However, this required the assumption of a limiting procedure which retains the four types of vector interaction terms in four dimensions. Without any such assumptions, the generic prediction is a viable massive vector field candidate for dark matter.

Acknowledgments: J.B.J. acknowledges the financial support of A*MIDEX project (n ANR-11-IDEX-0001-02) funded by the Investissements d'Avenir French Government program, managed by the French National Research Agency (ANR), MINECO (Spain) projects FIS2014-52837-P, FIS2016-78859-P (AEI/FEDER) and Consolider-Ingenio MULTIDARK CSD2009-00064

Author Contributions: J.B.J. and T.S.K. contributed equally to the paper.

Conflicts of Interest: The authors declare no conflict of interest.

\section{References}

1. Olmo, G.J. Palatini Approach to Modified Gravity: f(R) Theories and Beyond. Int. J. Mod. Phys. D 2011, 20, 413-462.

2. Capozziello, S.; Harko, T.; Koivisto, T.S.; Lobo, F.S.N.; Olmo, G.J. Hybrid metric-Palatini gravity. Universe 2015, 1, 199-238.

3. Hammond, R.T. Torsion gravity. Rep. Prog. Phys. 2002, 65, 599-649.

4. Krasnov, K. Non-Metric Gravity. I. Field Equations. Class. Quant. Gravity 2008, 25, 025001.

5. Sobreiro, R.F.; Vasquez Otoya, V.J. Aspects of nonmetricity in gravity theories. Braz. J. Phys. 2010, 40, $370-374$. 
6. Vitagliano, V. The role of nonmetricity in metric-affine theories of gravity. Class. Quant. Gravity 2014, 31, 045006.

7. Beltran Jimenez, J.; Golovnev, A.; Karciauskas, M.; Koivisto, T.S. The Bimetric variational principle for General Relativity. Phys. Rev. D 2012, 86, 084024

8. Scholz, E. The unexpected resurgence of Weyl geometry in late 20-th century physics. arXiv 2017, arXiv:1703.03187.

9. Tanhayi, M.R.; Dengiz, S.; Tekin, B. Weyl-Invariant Higher Curvature Gravity Theories in n Dimensions. Phys. Rev. D 2012, 85, 064016.

10. Haghani, Z.; Khosravi , N.; Shahidi, S. The Weyl-Cartan Gauss-Bonnet gravity. Class. Quant. Gravity2015, 32, 215016.

11. Beltran Jimenez, J.; Koivisto, T.S. Extended Gauss-Bonnet gravities in Weyl geometry. Class. Quant. Gravity 2014, 31, 135002

12. Beltran Jimenez, J.; Koivisto, T.S. Spacetimes with vector distortion: Inflation from generalised Weyl geometry. Phys. Lett. B 2016, 756, 400-404.

13. Hehl, F.W.; Macias, A. Metric affine gauge theory of gravity. 2. Exact solutions. Int. J. Mod. Phys. D 1999, 8, 399-416.

14. Kallosh, R.; Linde, A.; Roest, D. Superconformal Inflationary $\alpha$-Attractors. J. High Energy Phys. 2013, 2013, 198.

15. Ozkan, M.; Pang, Y.; Tsujikawa, S. Planck constraints on inflation in auxiliary vector modified $f(R)$ theories. Phys. Rev. D 2015, 92, 023530.

16. Ozkan, M.; Roest, D. Universality Classes of Scale Invariant Inflation. arXiv 2015, arXiv:1507.03603.

17. Akarsu, O.; Boran, S.; Kahya, E.O.; Ozdemir, N.; Ozkan, M. Broken Scale Invariance, Alpha-Attractors and Vector Impurity. arXiv 2016, arXiv:1606.05308.

18. Beltran Jimenez, J.; Heisenberg, L.; Koivisto, T.S. Cosmology for quadratic gravity in generalized Weyl geometry. J. Cosmol. Astropart. Phys. 2016, 2016, 046.

19. Cembranos, J.A.R.; Hallabrin, C.; Maroto, A.L.; Jareno, S.J.N. Isotropy theorem for cosmological vector fields. Phys. Rev. D 2012, 86, 021301.

20. Cembranos, J.A.R.; Maroto, A.L.; Jareno, S.J.N. Isotropy theorem for cosmological Yang-Mills theories.

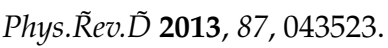

21. Cembranos, J.A.R.; Maroto, A.L.; Jareno, S.J.N. Isotropy theorem for arbitrary-spin cosmological fields. J. Cosmol. Astropart. Phys. 2014, 2014, 042.

22. Tasinato, G. Cosmic Acceleration from Abelian Symmetry Breaking. J. High Energy Phys. 2014, $2014,067$.

23. Heisenberg, L. Generalization of the Proca Action. J. Cosmol. Astropart. Phys 2014, $2014,015$.

24. Allys, E.; Peter, P.; Rodriguez, Y. Generalized Proca action for an Abelian vector field. J. Cosmol. Astropart. Phys. 2016, 2016, 004.

25. Beltran Jimenez, J.; Heisenberg, L. Derivative self-interactions for a massive vector field. Phys. Lett. B 2016 $757,405-411$.

26. Beltran Jimenez, J.; Maroto, A.L. Dark energy, non-minimal couplings and the origin of cosmic magnetic fields. J. Cosmol. Astropart. Phys. 2010, 2010, 025.

27. Beltran Jimenez, J.; Piazza, F.; Velten, H. Evading the Vainshtein Mechanism with Anomalous Gravitational Wave Speed: Constraints on Modified Gravity from Binary Pulsars. Phys. Rev. Lett. 2016, 116, 061101.

28. Horndeski, G.W. Conservation of Charge and the Einstein-Maxwell Field Equations. J. Math. Phys. 1976, 17, 1980-1987.

29. Barrow, J.D.; Thorsrud, M.; Yamamoto, K. Cosmologies in Horndeski's second-order vector-tensor theory. J. High Energy Phys. 2013, 2013, 146.

30. Beltran Jimenez, J.; Durrer, R.; Heisenberg, L.; Thorsrud, M. J. Cosmol. Astropart. Phys. 2013, 2013, 064.

(C) 2017 by the authors. Licensee MDPI, Basel, Switzerland. This article is an open access article distributed under the terms and conditions of the Creative Commons Attribution (CC BY) license (http:/ / creativecommons.org/licenses/by/4.0/). 